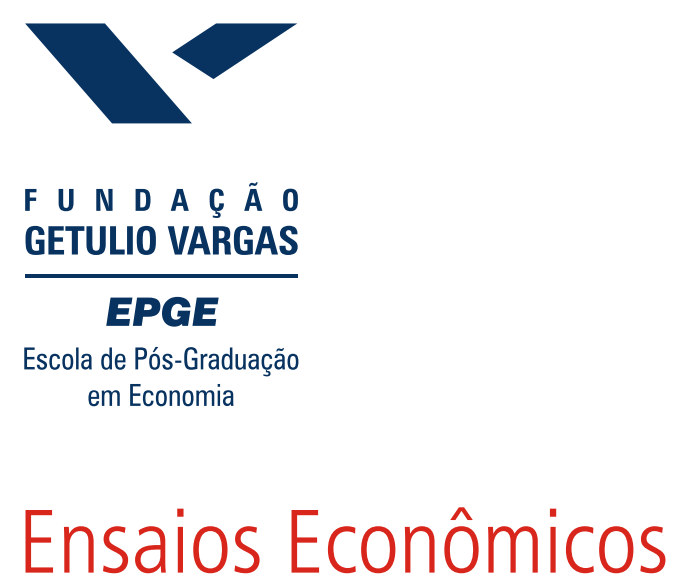

$$
\begin{aligned}
& \text { Escola de } \\
& \text { Pós Graduação } \\
& \text { em Economia } \\
& \text { da Fundação } \\
& \text { Getulio Vargas }
\end{aligned}
$$

$N^{\circ} 603$

ISSN 0104-8910

Some Benefits of Cyclical Monetary Policy

Ricardo de O. Cavalcanti, Ed Nosal

Outubro de 2005 
Os artigos publicados são de inteira responsabilidade de seus autores. As opiniões neles emitidas não exprimem, necessariamente, o ponto de vista da Fundação Getulio Vargas. 


\title{
Some Benefits of Cyclical Monetary Policy*
}

\author{
Ricardo de O. Cavalcanti ${ }^{\dagger}$ and Ed Nosal ${ }^{\ddagger}$
}

July 2003 (This version October 2005)

\begin{abstract}
In this paper, we present a simple random-matching model of seasons, where different seasons translate into different propensities to consume and produce. We find that the cyclical creation and destruction of money is beneficial for welfare under a wide variety of circumstances. Our model of seasons can be interpreted as providing support for the creation of the Federal Reserve System, with its mandate of supplying an elastic currency for the nation.
\end{abstract}

\section{Introduction}

Should monetary policy be cyclical? Although this is an old question in monetary economics, there is no general consensus as to the correct answer. Recent research on the "pure theory of money" has contributed very little, if anything, to the debate that surrounds this question. By pure theory of money, we refer to that line of research where money arises endogenously as a solution to a trading problem, instead of being treated as a primitive of the economic environment, such as preferences and technology. Perhaps it is not so surprising that modern theories of money have remained silent on the desirability of cyclical monetary policy; although the environments that are suitable for modeling a role for fiat money - environments with infinite horizons and diverse trading opportunities - are quite tractable when they are stationary, they become quite intractable when the stationarity assumption is relaxed. In this paper we explore a simple departure from the standard model of money as a medium of

${ }^{*}$ The views expressed here are those of the authors and not necessarily those of the Federal Reserve Bank of Cleveland or the Federal Reserve System.

${ }^{\dagger}$ Getulio Vargas Foundation, Brazil; ricardoc@fgv.br

${ }^{\ddagger}$ Federal Reserve Bank of Cleveland, U.S.A.; ed.nosal@clev.frb.org 
exchange; in particular, we construct a model with seasonal fluctuations in output, where money is essential and where the cyclical creation and destruction of money can be welfare enhancing.

We claim that cyclical policies ought to have a role when the standard model is generalized to account for seasonal movements in output and consumption. Our argument is twofold. First, because money is essential, there necessarily exist some sorts of frictions in the economy. But the very existence of these frictions mean that the standard welfare theorems will not apply. Hence, monetary economies will be relegated to the world of the second best. In a second best world, activist government policies may be beneficial. However, in the context a monetary environment, it should not be the case that activist government policies can implement the first-best allocation, for this would imply that government policies can somehow "neutralize" the fundamental frictions that exist in the economy. For example, monetary policy should not be able to overcome the fact that money holdings are a less-than-perfect substitute for credit. Because of this, a distribution of money holdings should persist after central bank interventions, so long as full insurance against idiosyncratic risk is unattainable. Second, and consequently, any monetary policy aimed at improving the efficiency of monetary trades ought to attempt to make adjustments in the distribution of money over the economic cycles. In this paper we study a simple framework in which a beneficial role for cyclical monetary policy is derived, and we believe that this result will remain valid for any generalization to the model environment which preserves the second-best aspect of fiat money.

The task that we set for ourselves is to construct a model where the social role of money varies over a cycle and where the monetary authority can "react" to the cyclical nature of money by using a limited set of policy instruments, namely lumpsum creation and destruction. As well, we want to maintain a reasonably sized, i.e., small, state space for the economy. We find that a simple alternating movement in preferences, studied from the perspective of mechanism design, within a set of cyclical but otherwise stationary allocations, can be addressed without difficulties when money holdings are limited to either zero or one unit. We wish to emphasize that the limited holdings of money are used for analytical tractability and do not 
drive the key results.

The model, the creation of the Fed, and some literature

In our model economy, individual agents experience seasonal preference shocks and trade between pairs of agents is characterized by a lack of double coincidence of wants. Agents in the economy belong to one of two equally sized groups. When one group has a production opportunity the other group has a consumption opportunity and, on a period by period basis, each group alternates between having production and consumption opportunities. In pairwise meetings, the consumer faces an idiosyncratic preference shock which affects his desire to consume. The notion of seasons is introduced by having the (economy wide) distribution of consumer preference shocks differ over even and odd dates. For example, the even period will be a high demand season and the odd period a low demand season if the total number of consumer agents who actually want to consume in even periods is greater than the total number of consumer agents who want to consume in odd periods.

Monetary policy is restricted to take the form of a reoccurring pattern of taxing money holdings in one period and injecting the proceeds in a lump-sum fashion in the subsequent period. If taxes and subsidies are non-zero, then monetary policy will be cyclical; if taxes and subsidies are equal to zero, then the money supply will be constant. We first show that under a constant monetary policy rule, the seasonal frequency of trade is constant. When we compare a cyclical monetary policy with a constant money supply policy we find two basic effects. First, cyclical policies may reduce the return on money and, hence, reduce the producers' desire to supply output. This effect, the so-called intensive margin effect, may reduce the social surplus associated with each trade. Second, if there is a sufficient asymmetry in the distribution of aggregate preference shocks, so that one season has a relatively high desire or demand for consumption compared to the other season, then a cyclical monetary policy will increase the average frequency of trades, or the extensive margin, compared to a constant money supply policy. We find that under a wide variety of circumstances, the optimal monetary policy will be cyclical. So, although a cyclical monetary policy may result in a lower and inefficient level of production at the match level, the fact that the economy wide frequency of trades increases implies that a 
cyclical monetary policy can increase the welfare of society, when compared to a constant money supply policy.

The results from our model can be loosely be interpreted as providing some support for the creation of the Federal Reserve System in 1913. The preamble to the Federal Reserve Act states that the Federal reserve banks were established to, among other things, "furnish an elastic currency." According to Meltzer (2003) there are two meanings for the word "elasticity." One meaning is in regard to the ability of a central bank to pool reserves and lend them out in the event of a banking or financial crisis. The second meaning is in reference to seasonal fluctuations, which is the topic of this paper. In practice, the two meanings of elasticity are not unrelated because the data indicates that seasonal fluctuations in money demand can exacerbate a (potential) banking or financial panic. For example, farmers needed cash in the autumn in order to harvest their crops but, given the structure of the banking system before the founding of the Fed, there was essentially only a fixed amount of reserves to go around. As a result, the increase in demand for cash in the autumn could potentially turn a quite independent and manageable liquidity problem in financial markets into a financial panic or banking crisis.

Miron (1986) concludes that the founding of the Fed had positive welfare consequences for the economy since its policy of furnishing an elastic currency greatly reduced the possibility of financial panics. In particular, financial panics were commonplace and sometimes quite severe before the founding of the Fed and this was no longer so after the founding. Note that the two meanings of elasticity are at play here. By consolidating reserves at a central place, the Fed was able to provide reserves to banks who needed them in a time of financial stringency. Furthermore, by discounting real bills, the Fed was able to provide (additional) liquidity to farmers, implying that their increase in demand for money need not exacerbate a potential liquidity problem in financial markets. Miron (1986) points out, however, if an economy has deposit insurance, then an elastic currency policy would not be welfare improving since existence of deposit insurance would greatly reduce, if not eliminate, the possibility of financial panics, which is the source of the welfare gain in his analysis. In this paper, we completely abstract any notion of financial panics and find that there 
are other sources for welfare gains associated with an elastic currency (cyclical monetary) policy and that is the provision of an elastic currency can increase the average frequency of trade in the economy.

Since the "fine tuning" of monetary policy is a broad topic with voluminous contributions, it is important to relate our model to some well known papers up front, so as to highlight the particular debt our work owes to this broad literature. Lucas (1972) was the first to present a pure theory of short-run effects of monetary policy, but an important ingredient in his analysis is an exogenous and random supply of money. In a competitive environment, the optimal monetary policy invariably leads to the Friedman (1969) rule in the form of a deflation that eliminates the opportunity cost of holding money. Bewley (1980), Levine (1991), and Sheinkman and Weiss (1986), among others, departed from a representative consumer structure and found that there exist welfare gains associated with an ongoing inflation. In these models, traders face uninsurable shocks and can benefit from some redistribution of wealth generated by inflation. The literature that has followed Kiyotaki and Wright (1989) and their seminal work on the media of exchange, has more or less been limited to reproducing these inflation gains. ${ }^{1}$

The rest of the paper is divided as follows. In section 2, we describe the environment with two seasons. In section 3, we define symmetric and stationary allocations, as well as the welfare criteria that guides the discussion of optimal monetary policies. In section 4 , we define what we mean by an implementable allocation. Section 5 analyzes extensive margin effects associated with a cycle monetary policy while section 6 analyzes intensive margin effects. Section 7 characterizes the optimal monetary policy and section 8 concludes.

\section{The environment}

Time is discrete and the horizon is infinite. There are 2 types of people, each of which is defined on a $[0,1]$ continuum. Each type of person is specialized in consumption

\footnotetext{
${ }^{1}$ See Molico (1999), and Deviatov and Wallace (2001). There has been also work on the effects of inflation on search intensity, such as Li (1995) and Shi (1999), among others.
} 
and production: A type $e$ person consumes even-date goods and produces odd-date goods while a type $d$ person consumes odd-date goods and produces even-date goods. We find it convenient to refer to a type $e$ individual in an even (odd) date, or a type $d$ individual in an odd (even) date, as a consumer (producer). Each type of person maximizes expected discounted utility, with a common discount factor $\beta \in(0,1)$. Let $s \in\{e, d\}$ indicate the season and/or the type of person. We find it useful to have a notation for the two-period discount factor, $\delta \equiv \beta^{2}$.

The utility function for a consumer in season $s \in\{e, d\}$ is $\varepsilon_{s} u_{s}\left(y_{s}\right)$, where $\varepsilon_{s}$ is the idiosyncratic shock affecting this consumer and $y_{s} \in R_{+}$is the amount consumed. The shock $\varepsilon_{s}$ is Bernoulli and the probability that $\varepsilon_{s}=1, \pi_{s} \in(0,1)$, is indexed by the season $s$. A producer in season $s$ can produce any choice of $y_{s} \geq 0$ at a utility cost normalized to be $y_{s}$ itself. Utility in a period is thus $\varepsilon_{s} u_{s}\left(y_{s}\right)$ when consuming, and $-y_{s}$ when producing. The function $u_{s}$ is assumed to be increasing, twice differentiable, and satisfies $u_{s}(0)=0, u_{s}^{\prime \prime}<0, u_{s}^{\prime}(0)=\infty$ and $u_{s}^{\prime}(\infty)<1$. We assume that $\pi_{e} \geq \pi_{d}$ and $u_{e}^{\prime} \geq u_{d}^{\prime}$ so that even dates feature a high desire for consumption-both at the individual and aggregate levels - relative to odd dates. It should be emphasized that a strict inequality for either these gives rise to a cyclical demand for liquidity.

In every period a type $e$ person is matched randomly with a type $d$ person. During meetings, the realization of preference shocks occurs and production may take place.

All individuals are anonymous, in the sense that they all have private histories. We also assume that people cannot commit to future actions, so that those who produce have to get a tangible (future) reward for doing so. The reward, in this paper, takes the form of fiat money. To keep the model simple, we assume that each person can carry from one meeting to the next either 0 or 1 units of fiat money. A consequence of this assumption, which makes the distribution of people tractable, is that trade will only take place when the consumer realizes $\varepsilon_{s}=1$ and has money, and the producer has no money.

Monetary policy takes the simple form of a choice of the pair $(\sigma, \tau)$, where $\sigma$ is the probability that a person without money gets one unit of money before meetings, and $\tau$ is the probability that a person with money loses the money before meetings. Let $M_{e}$ denote the measure of individuals holding money in even periods and $M_{d}$ the 
measure of individuals holding money in odd periods. We restrict attention to cases in which either $\tau=\sigma=0$ in all dates, or $\sigma>0$ in even dates and $\tau>0$ in odd dates. This simple formulation is designed to limit our analysis to the specific question of whether periods of high desire for consumption should experience an increase in the supply of money, which is offset by a reduction of economy wide money balances in the subsequent period.

\section{$3 \quad$ Stationarity and welfare criteria}

We let the measure of consumers with money during meetings in season $s$ be denoted by $q_{s}$ and consumers without money denoted by $1-q_{s}$. We let the measure of producers without money during meetings in season $s$ be denoted $p_{s}$ and producers with money denoted by $1-p_{s}$. In order to save on notation, let $y=\left(y_{e}, y_{d}\right)$ denote the list of output levels, let $x \equiv\left(p_{e}, q_{e}, p_{d}, q_{d}\right)$ denote an arbitrary distribution, and use the superscript + , as in $x^{+} \equiv\left(p_{e}^{+}, q_{e}^{+}, p_{d}^{+}, q_{d}^{+}\right)$, when the qualification that $\sigma>0$ for that distribution becomes essential. A distribution $x \in[0,1]^{4}$ is considered invariant if and only if there exists $(\sigma, \tau) \in[0,1]^{2}$ such that

$$
\begin{gathered}
p_{e}=(1-\sigma)\left(1-q_{d}+\pi_{d} p_{d} q_{d}\right), \\
p_{d}=(1-\tau)\left(1-q_{e}+\pi_{e} p_{e} q_{e}\right)+\tau, \\
q_{e}=(1-\sigma)\left(1-p_{d}+\pi_{d} p_{d} q_{d}\right)+\sigma
\end{gathered}
$$

and

$$
q_{d}=(1-\tau)\left(1-p_{e}+\pi_{e} p_{e} q_{e}\right)
$$

where the distribution $x$ is described after money is created or destroyed.

The stationarity requirement (1) can be explained as follows. During odd-date meetings, trade takes place after the destruction of money. The measure of consumers with money is $q_{d}$ and the measure of producers without money is $p_{d}$. Consumers without money, whose number is $1-q_{d}$, cannot buy goods; each of them faces a probability $\sigma$ of finding money at the beginning of the next date. Hence, $1-\sigma$ times $1-q_{d}$ is the total flow of those consumers who become producers without money in 
the next (even) date. Similarly, the measure of consumers with money in the odd date is $q_{d}$. Only a fraction $\pi_{d}$ of these consumers will want to consume in the odd date and only a fraction $q_{d}$ of these consumers will meet a producer without money. Therefore, $\pi_{d} p_{d} q_{d}$ represents the measure of consumers with money that will trade in date $d$ and $(1-\sigma) \pi_{d} p_{d} q_{d}$ represents the number of these consumers that become producers without money in the next (even) period, after money creation takes place.

Likewise, regarding requirement (2), we first notice that a measure $1-q_{e}+\pi_{e} p_{e} q_{e}$ producers arrive at the beginning of date $d$ without money. Adding now to that the mass of money destroyed from those date $e$ consumers with money who did not trade, $\tau q_{e}\left(1-\pi_{e} p_{e}\right)$, yields the right-hand of $(2)$. The same principle explains requirement (3). The measure of consumers with money at date $e$ consists of the measure of producers who leave date $d$ with money, $1-p_{d}+\pi_{d} p_{d} q_{d}$, and the measure of producers who leave date $d$ without money but obtain some when additional money is created at the beginning of date $e, \sigma p_{d}\left(1-\pi_{d} q_{d}\right)$. Finally, requirement (4) follows from imposing stationarity on the measure of consumers with money arriving at date $d, 1-p_{e}+\pi_{e} p_{e} q_{e}$, after the destruction of money takes place with probability $\tau$.

Our notion of stationarity amounts to restricting that output, $y_{s}$, as well as the measures $p_{s}$ and $q_{s}$, to be constant functions of the season, $s$, only. These functions are used symmetrically in a measure of welfare as follows. We adopt an ex ante welfare criteria, with an expected discounted utility computed according to an invariant distribution and output function. Whenever trade takes place in a season, it is because money is changing hands from a fraction, $p_{s}$, of the mass of consumers $\pi_{s} q_{s}$ in position to trade. Since, for each consumer there is a producer, the flow of total utility in season $s$ is $\pi_{s} p_{s} q_{s}\left[u_{s}\left(y_{s}\right)-y_{s}\right]$. We call the term $\pi_{s} p_{s} q_{s}$ the extensive margin at $s$, and $u_{s}\left(y_{s}\right)-y_{s}$ the intensive margin at $s$. The extensive margin is a property of the distribution $x$, while the intensive margin is a property of outputs $y$. An allocation is a pair $(x, y)$, where $x$ and $y$ are invariant and $y$ has nonnegative coordinates. The welfare $U$ attained by an allocation is defined as the present discounted value

$$
U(x, y)=\frac{1}{(1-\beta)} \sum_{s} \pi_{s} p_{s} q_{s}\left[u_{s}\left(y_{s}\right)-y_{s}\right]
$$

The intensive margin at $s$ is maximized at $y_{s}^{*}$, where $u_{s}^{\prime}\left(y_{s}^{*}\right)=1$, which is uniquely 
defined by assumption. We refer to $y^{*}=\left(y_{e}^{*}, y_{d}^{*}\right)$ as the first-best output list.

\section{Implementable Allocations}

The definition of the values of $y$ consistent with incentive compatibility follows the notion of sequential individual rationality employed by Cavalcanti and Wallace (1999), and also applied by Cavalcanti (2004). Underlying their definition of participation constraints is the idea that a social planner proposes an allocation, but that anonymous individuals may defect from that proposal by not trading in a given meeting. If individual(s) defect, then they do not lose any money holdings that were brought into the meeting. We adopt the same concept here, with the exception to the taxation of money holdings, which we assume cannot be avoided by individuals with money. The participation constraints are then defined by a set of allocations, according to the expected discounted utilities implied by the allocations. In order to be able to represent these constraints, we need first to describe the Bellman equations of the economy.

The value functions will be computed before the realization of the effects of creation and destruction of money for each individual in a given date. (Recall that money is created at the beginning of even dates and is destroyed at the beginning of odd dates.) The value function for consumers with money at $s$ is $v_{s}$, and that for producers without money is $w_{s}$. We define $\bar{v}_{s}$ as the value for consumers without money at $s$, and $\bar{w}_{s}$ as that of producers with money. The Bellman equations for $(v, w)=\left(v_{e}, v_{d}, w_{e}, w_{d}\right)$ are defined by

$$
\begin{aligned}
v_{e} & =\pi_{e} p_{e}\left(u_{e}+\beta w_{d}\right)+\left(1-\pi_{e} p_{e}\right) \beta \bar{w}_{d} \\
w_{e} & =\sigma \beta v_{d}+(1-\sigma)\left[\pi_{e} q_{e}\left(-y_{e}+\beta v_{d}\right)+\left(1-\pi_{e} q_{e}\right) \beta \bar{v}_{d}\right] \\
v_{d} & =\tau \beta w_{e}+(1-\tau)\left[\pi_{d} p_{d}\left(u_{d}+\beta w_{e}\right)+\left(1-\pi_{d} p_{d}\right) \beta \bar{w}_{e}\right] \\
w_{d} & =\pi_{d} q_{d}\left(-y_{d}+\beta v_{e}\right)+\left(1-\pi_{d} q_{d}\right) \beta \bar{v}_{e}
\end{aligned}
$$

where $u_{e}$ and $u_{d}$, by an abuse of notation, stand for $u_{e}\left(y_{e}\right)$ and $u_{d}\left(y_{d}\right)$, respectively. 
The definition is completed by substituting for the values of $(\bar{v}, \bar{w})$, given by,

$$
\begin{aligned}
\bar{v}_{e} & =\sigma v_{e}+(1-\sigma) \beta w_{d} \\
\bar{w}_{e} & =\beta v_{d} \\
\bar{v}_{d} & =\beta w_{e} \\
\bar{w}_{d} & =\tau w_{d}+(1-\tau) \beta v_{e}
\end{aligned}
$$

into the previous system.

The participation constraint for producers at even dates is simply

$$
-y_{e}+\beta v_{d} \geq \beta \bar{v}_{d}=\delta w_{e}
$$

since an even date producer is bringing no money into a meeting, and only has the option of leaving the meeting and becoming a producer two periods later. Producers at odd dates have to take into account that if they disagree with producing the planned output $y_{d}$ and walk away from a trade, then they have a chance of receiving money next period from the money-creation policy. Thus, the participation constraint for producers at odd dates can be stated as

$$
-y_{d}+\beta v_{e} \geq \beta \bar{v}_{e}=\beta \sigma v_{e}+\delta(1-\sigma) w_{d}
$$

For completeness, we state the participation constraint for consumers, which can be shown to be implied by the participation constraints of producers. They are

$$
u_{e}+\beta w_{d} \geq \beta \bar{w}_{d}
$$

and

$$
u_{d}+\beta w_{e} \geq \beta \bar{w}_{e}
$$

An allocation $(x, y)$ is said to be implementable if $x \equiv\left(p_{e}, q_{e}, p_{d}, q_{d}\right)$ is invariant for some policy $(\sigma, \tau)$ such that there exists $(v, w)$ and $(\bar{v}, \bar{w})$ for which (5)-(10) hold. An allocation is to be optimal if it maximizes $U(x, y)$ among the set of implementable allocations. 


\section{$5 \quad$ Extensive-margin effects}

Monetary policy can be viewed as a choice of an invariant distribution $x$. Changes in $x$ due to changes in $(\sigma, \tau)$ have direct effects on extensive margins, $\pi_{s} p_{s} q_{s}$, and indirect effects on intensive margins, $u_{s}\left(y_{s}\right)-y_{s}$, through the participation constraints, i.e., $y$ depends on $x$. Note that the latter effects can be ignored if for both $s=e$ and $s=d$, the maximizer of $u_{s}\left(y_{s}\right)-y_{s}, y_{s}^{*}$, satisfies participation constraints. In this section, we investigate whether the maximizer of the sum $\sum_{s} \pi_{s} p_{s} q_{s}$, among all invariant distributions $x$, is a cyclical policy $x^{+}$, that is, one with a positive $\sigma$. We shall see that a cyclical monetary policy tends to increase the extensive margin at $e$, and to decrease that at $d$. Since $u_{e}^{\prime} \geq u_{d}^{\prime}$, it will follow that if $y^{*}$ satisfies participation constraints and the maximizer of the sum $\sum_{s} \pi_{s} p_{s} q_{s}$ is cyclical, then the optimal allocation is indeed a cyclical monetary policy.

Acyclical Distributions We start by pointing out an important property of the invariant distributions when the money supply is constant, i.e., when $\sigma=0$. If $x$ is invariant when $\sigma=0$, we will say that $x$ is acyclical, a label motivated by the following lemma.

Lemma 1 Assume that $x$ is acyclical. Then the extensive margin, $\pi_{s} p_{s} q_{s}$, is constant in $s$.

Proof. Set $\sigma=\tau=0$ in equations (1) and (4). It follows that $\pi_{e} p_{e} q_{e}=\pi_{d} p_{d} q_{d}$.

Interestingly, the property of constant extensive margins holds regardless of the relative values of $\pi_{s}$. We can offer an intuitive explanation for this property as follows. Let us consider the inflow and outflow of money for a set of individuals of the same type, say type $e$. Then, on one hand, the stationary measure of consumers of this type spending money is $\left(\pi_{e} p_{e}\right) q_{e}$, an event taking place at even dates. On the other hand, the stationary measure of producers of this type acquiring money is $\left(\pi_{d} q_{d}\right) p_{d}$, an event taking place at odd dates. Since the quantity of money in the hands of this group must be stationary, and all seasons have the same frequency, then these two margins must be equalized, as stated in the lemma. 
Some useful observations about acyclical distributions can be made with regard to the relative values of $p_{s}$ and $q_{s}$.

Lemma 2 Assume that $x$ is acyclical. Then (i) $p_{e}-q_{e}=p_{d}-q_{d}$, and (ii) $p_{e} \leq p_{d}$ if and only if $\pi_{d} \leq \pi_{e}$.

Proof. (i) Set $\sigma=\tau=0$ in equations (1) and (2). Since, by lemma 1, $\pi_{e} p_{e} q_{e}=$ $\pi_{d} p_{d} q_{d}$, equations (1) and (2) imply that $p_{e}-q_{e}=p_{d}-q_{d}$. (ii) Since, by lemma 1 , $\pi_{e} p_{e} q_{e}=\pi_{d} p_{d} q_{d}, \pi_{e} \geq \pi_{d}$ if and only if $p_{e} q_{e} \leq p_{d} q_{d}$. Part (i) of this lemma implies that if $p_{e} q_{e} \leq p_{d} q_{d}$, then $p_{e} \leq p_{d}$ and $q_{e} \leq p_{d}$.

There is an alternative way to think about part (i) of lemma 1. The measure of individuals that hold money in period $s, M_{s}$, is the sum of consumers with money, $q_{s}$, and producers with money, $1-p_{s}$. When $\sigma=\tau=0$, the measures of individuals that hold money in odd and even periods are the same, i.e., $M_{e}=M_{d}$, which implies that $1-p_{e}+q_{e}=1-p_{d}+q_{d}$, or that $p_{e}-q_{e}=p_{d}-q_{d}$.

An application of lemma 2 allows us to describe the set of acyclical distributions when $\pi_{e}=\pi_{d}$ in rather simple terms.

Lemma 3 Assume $\pi_{e}=\pi_{d}=\pi$. Then the set of acyclical distributions is fully described by $p_{e}=p_{d}=p, q_{e}=q_{d}=q$, and $p=1-q+\pi p q$ for $q \in[0,1]$.

Proof. Since $\pi_{e}=\pi_{d}$ then, by lemma $2, p_{e}=p_{d}$, and consequently, by lemma 1 , $q_{e}=q_{d}$. Equation (1) with $\sigma=0$ thus proves the lemma.

The one-dimensional set described by lemma 3 is the symmetric set of distributions that appears in Cavalcanti (2004). The equation $p=1-q+\pi p q$ defines a strictly concave function for $q \in[0,1]$, and the extensive margin $\pi p q$ is maximized when $p=q=\left[1-(1-\pi)^{\frac{1}{2}}\right] / \pi$.

Properties similar to those described by lemma 3 also obtain when $\pi_{e}>\pi_{d}$; for example, every acyclical $x$ can be indexed by a one-dimensional choice of $q_{d}$,

Lemma 4 When $\pi_{e}>\pi_{d}$ there exists, for each $q_{s}$, an unique acyclical $x$. Moreover, $x$ can be solved for analytically. The statement holds for any $s$ in $\{e, d\}$.

Proof. See appendix 1. 
And, the extensive margin is maximized when the measure of consumers with money equals the measure of producers without money,

Proposition 1 When $\pi_{e}>\pi_{d}$ the maximizer of $\pi_{s} p_{s} q_{s}$, among the set of acyclical distributions, is the unique $x$ such that $p_{s}=q_{s}$ for $s \in\{e, d\}$.

Proof. See appendix 2 .

Hence, when the money supply is constant, the distribution that maximizes the extensive margin is characterized by $p_{d}=q_{d}$ and $p_{e}=q_{e}$. This result echoes a standard result in many search models of money, which is that it is optimal for half of the population to hold money. Such a distribution of money holdings maximizes the number of productive matches. To see that our model also has this feature, note that when $\sigma=\tau=0$ and when $\pi_{s} p_{s} q_{s}$ is maximized, i.e., $p_{s}=q_{s}$ for $s \in\{e, d\}$, then measure of individuals holding money at date $s$ is $1-p_{s}+q_{s}=1$. Since the total measure of individuals in the economy is 2 , having half the population holding money maximizes the extensive margin when $\sigma=\tau=0$. Note that value of $x$ is easily computed when the extensive margin is maximized,

Lemma 5 If $x$ is acyclical and $p_{s}=q_{s}$, then

$$
p_{d}=\frac{1+\sqrt{\theta}-\sqrt{(1+\sqrt{\theta})^{2}-4 \pi_{d}}}{2 \pi_{d}},
$$

and

$$
p_{e}=1-p_{d}+\pi_{d} p_{d}^{2}
$$

where $\theta=\pi_{d} / \pi_{e}$.

Proof. Since by lemma $1, \pi_{e} p_{e} q_{e}=\pi_{d} p_{d} q_{d}$, equation (2) with $\tau=0$ yields $p_{d}=$ $1-q_{e}+\pi_{e} p_{e} q_{e}$. Because $q_{s}=p_{s}$, then $p_{e}=\sqrt{\theta} p_{d}$ and $p_{d}=1-p_{e}+\pi_{d} p_{d}^{2}$. The last two expressions yields a quadratic equation in $p_{d}$ whose only relevant solution is as stated. The value for $p_{e}$ can be computed from the last expression once $p_{d}$ is determined.

This completes our discussion about acyclical distributions, i.e., about a constant money supply. We can now move the discussion on to cyclical money policy and cyclical distributions. 
Cyclical Distributions We now consider small pertubations in the quantity of money. We consider cyclical distributions $x^{+}$in a neighborhood of a given acyclical $x$. Our ultimate goal is to describe and sign the derivative of the sum $\sum_{s} \pi_{s} p_{s} q_{s}$ with respect to $\sigma$, evaluated at $\sigma=0$ and $p_{s}=q_{s}$. It follows, by force of proposition 1 , that if this derivative is positive, then the maximizer of the sum must be cyclical. Clearly, the system (1)-(4) that defines $x^{+}$depends on $\sigma$ and $\tau$. The existence of $x^{+}$ follows from a simple fixed-point argument.

Lemma 6 Let $(\tau, \sigma) \in(0,1)^{2}$ be fixed. Then there exists an invariant distribution $x^{+}$.

Proof. The right-hand side of (1)-(4) defines a continuous function of $x^{+}$, with domain on the compact and convex set $[0,1]^{4}$. The result then follows from Brower's fixed point theorem.

If $x^{+}$is invariant, then the quantity of money destroyed in season $d$ must equal the quantity created in $e$, that is,

$$
\tau\left(1-p_{e}^{+}+q_{e}^{+}\right)=\sigma\left(1-q_{d}^{+}+p_{d}^{+}\right)
$$

It can be shown that the equality (11) is implied by the system (1)-(4). The quantity of money during season $e$ meetings, just before trade, is given by the mass $1-p_{e}^{+}$ with producers, plus the mass $q_{e}^{+}$with consumers. Since trade itself does change this quantity of money, and each money holder at the beginning of next season faces a probability $\tau$ of losing his money, then the total amount of money destroyed is given by the left-hand side of (11). Likewise, the measure of individuals without money at the end of season $d$ is $1-q_{d}^{+}+p_{d}^{+}$, and since each of those finds money at the beginning of season $e$ with probability $\sigma$, then the quantity of money created is that expressed in the right-hand side of (11).

While there is a continuum of acyclical distributions, i.e., when $\sigma=\tau=0$, one $x$ for each $q_{d}$ (lemma 4), the same does not hold for cyclical distributions. When $\sigma$ and $\tau$ are strictly positive, there is an inflow of money that has to be matched by an outflow of the same quantity. Our numerical experiments indicate that only one level of $q_{d}^{+}$produces quantities of money that is capable of equalizing inflows and outflows 
for a given pair $(\sigma, \tau)$. We can, however, pin down the neighborhood in which $q_{d}^{+}$lies as follows. Because we want to associate $x^{+}$to a given $x$, we find it useful to define the constant $\phi$ with the property that, for $\tau=\phi \sigma, x^{+}$converges to $x$ as $\sigma$ approaches zero. Since the pair $(\sigma, \tau)$ must be consistent with the stationary quantities of money in the economy, expressed above by equation (11), the desired ratio of $\tau$ to $\sigma$, for a given $x=\left(p_{e}, q_{e}, p_{d}, q_{d}\right)$, is

$$
\phi=\frac{1-q_{d}+p_{d}}{1-p_{e}+q_{e}}
$$

By lemma 1 and proposition 1, the maximizer of the sum $\sum_{s} \pi_{s} p_{s} q_{s}$ among the set of acyclical distributions is the unique $x$ for which $\phi=1$. We assess the effects of pertubations by differentiating the system (1)-(4) with respect to $\sigma$ for $\phi$ fixed.

The lemma can be viewed as generalizing lemma 2 ; in words, the difference between the measures of consumers with money and producers without money will be equalized between seasons only if the distribution is acyclical,

Lemma 7 If $x$ is invariant and $\tau=\phi \sigma$, then $p_{s}-q_{s}=f_{s}(\sigma)$, where $f_{e}(\alpha)=\frac{\phi-\phi \alpha-1}{1+\phi-\phi \alpha}$ and $f_{d}(\alpha)=\frac{\phi+\phi \alpha-1}{1+\phi-\phi \alpha}$.

Proof. See appendix 3.

Note that $f_{s}$ does not depend on the fraction of consumers who desire to consume in season $s, \pi_{s}$.

The next proposition, which is the main result of this section, characterizes the sign of the derivative of the sum $\sum_{s} \pi_{s} p_{s} q_{s}$, evaluated at $p_{s}=q_{s}$ and $\sigma=0$ (the latter two equalities characterize the optimal constant money supply policy).

Proposition 2 The maximizer of sum $\sum_{s} \pi_{s} p_{s} q_{s}$ is cyclical if and only if $\pi_{d} \in[0, \bar{\pi}]$, where $\bar{\pi} \in\left(0, \pi_{e}\right)$ can be solved for analytically as a function of $\pi_{e}$.

Proof. See appendix 4.

The maximizer is therefore acyclical if $\pi_{d}=\pi_{e}$. Intuition behind which policyacyclical or cyclical - maximizes the average extensive margin is straightforward. Suppose that $\pi_{d}=\pi_{e}=\pi$. Then, the policy that maximizes the average extensive margin, is given by $p_{e}=p_{d}=q_{e}=q_{d} \equiv t$. Now if $\sigma$ is slightly increased from zero, there will be a stationary cyclical distribution $x^{+}$in the neighborhood of $x$. When $\sigma>0$, 
then $M_{d}=1-p_{d}^{+}+q_{d}^{+}<1<M_{e}=1-p_{e}^{+}+q_{e}^{+}$. Hence, it must be the case that $q_{e}$ increases by more than $p_{e}$ decreases and $q_{d}$ decreases by more than $p_{d}$ increases when $\sigma$ (and $\tau$ ) is increased from zero. Therefore, $p_{e}^{+} q_{e}^{+}>t^{2}$ and $p_{d}^{+} q_{d}^{+}<t^{2}$ : For a cyclical monetary policy, the extensive margin will increase in season $e$ and will decrease in season $d$, compared to the acyclical policy. Since, in a world with "no seasons," i.e., when $\pi_{d}=\pi_{e}=\pi$, a constant stock of money is optimal, it must be the case that the negative extensive margin effect associated with season $d$ outweighs the positive extensive margin effect associated with season $e$. Another way of thinking about this result is that when $p_{s} q_{s}$ is "equally weighted", i.e., $\pi_{e}=\pi_{d}$, the (negative) odd season effect dominates the (positive) even season effect. Suppose now that $\pi_{d}<\pi_{e}$. It will still be the case that $p_{e}^{+} q_{e}^{+}>p_{e} q_{e}$ and $p_{d}^{+} q_{d}^{+}<p_{d} q_{d}$, where $\left(p_{e}, q_{e}, p_{d}, q_{d}\right)$ is the distribution associated with the optimal acyclical monetary policy. However, since the differences between " $p$ " and " $q$ " do not depend upon $\pi_{e}$ and $\pi_{d}$, see lemma 7 , it may now be the case that the (positive) even season effect dominates the (negative) odd season effect. And this is because the even season matching probability of a consumer with money meeting a producer without money, $p_{e} q_{e}$, is weighted more heavily than the odd season matching probability, $p_{d} q_{d}$, i.e., $\pi_{e}>\pi_{d}$. Hence, if the fraction of potential consumers in odd periods is sufficiently smaller than the fraction of potential consumers in even periods - or if demand in the "high" season is sufficiently greater than demand in the "low" season, then a cyclical monetary policy will deliver a higher average extensive margin than the optimal acyclical policy.

\section{Intensive-margin effects}

The only participation constraints that are relevant, given our notion of stationarity, are those of producers. In this section, we derive representations of producer constraints as functions of preference parameters, policy parameters, and allocations, without references to value functions. While the first order effect of cyclical interventions is a tightening of participation constraints, these negative effects can be negligible or even absent if the discount factor is sufficiently high. 
Proposition 3 The participation constraints are satisfied if and only if

$$
u_{d}\left(y_{d}\right) \geq \frac{y_{e}}{\beta}\left[\frac{1}{(1-\tau) \pi_{d} p_{d}}-(1-\sigma) \delta \frac{1-\pi_{d} p_{d}}{\pi_{d} p_{d}}\right]
$$

and

$$
u_{e}\left(y_{e}\right) \geq \frac{y_{d}}{\beta}\left[\frac{1}{(1-\sigma) \pi_{e} p_{e}}-(1-\tau) \delta \frac{1-\pi_{e} p_{e}}{\pi_{e} p_{e}}\right] .
$$

Proof. See appendix 5.

Inequalities (13) and (14) indicate that cyclical policies have a potentially negative effect on intensive margins, since the right-hand side of both inequalities is increasing in $\sigma$ and $\tau$. The intuition behind these potential negative effects is straightforward: In either case - whether money is injected or withdrawn from the economy - the value of money in a trade will fall compared to the situation where $\sigma=\tau=0$. In the case where the money supply is contracted after production and trade, the value of currency falls because there is a chance that the producer will be unable to use his unit of currency in a future trade because it will be taken away; in the case where the money supply is expanded after production and trade, the fact that a producer may receive a unit of currency if he does not produce reduces the value of a unit of currency for a producer who does. A fall in the value of money implies that the amount of output received per unit of currency is reduced. If, however, $\beta$ is sufficiently high, then inequalities (13) and (14) will not bind at $y=y^{*}$, the output levels that maximize the intensive margins, and hence the potential effects on the intensive margins do not materialize for small monetary interventions.

Suppose that neither participation constraint binds when $\sigma=0$. Then, it turns out that if $\beta$ is reduced, the first participation constraint to be violated is the participation constraint for date-e producer, (13). Hence,

Lemma 10 If the participation constraint for date-e producers is satisfied for $x$ acyclical and $y=y^{*}$, then $(x, y)$ is implementable.

Proof. Since $u_{e}^{\prime} \geq u_{d}^{\prime}$ and $u_{e}(0)=u_{d}(0)$ then $u_{e}^{\prime}\left(y_{d}^{*}\right) \geq 1$, so that $y_{e}^{*} \geq y_{d}^{*}$ and $u_{e}^{*}\left(y_{e}^{*}\right) \geq u_{d}^{*}\left(y_{d}^{*}\right)$. Now, it has been established in the previous section that, if $x$ is acyclical, then $\pi_{d} \leq \pi_{e}$ implies $q_{d} \leq q_{e}$. As a result, the equality $\pi_{e} p_{e} q_{e}=\pi_{d} p_{d} q_{d}$, 
which holds for $x$, and $\pi_{d} \leq \pi_{e}$ implies $\pi_{d} p_{d} \leq \pi_{e} p_{e}$. Since the right-hand side of (13) or (14) is increasing in $\pi_{s} p_{s}$, and since $u_{e}^{*}\left(y_{e}^{*}\right) / y_{d}^{*} \geq u_{d}^{*}\left(y_{d}^{*}\right) / y_{e}^{*}$, then the result follows.

Lemma 10 indicates that it suffices to look at the participation constraint for date- $e$ producers in order to find a value of $\beta$ such that small interventions have no negative effects on intensive margins; the following proposition characterizes the critical $\beta$ for the optimal acyclical distribution such that the participation constraint for the date- $e$ "just" binds.

Proposition 3 Let $x$ take the value of the acyclical distribution with $p_{s}=q_{s}$, and let $\beta>\bar{\beta}$, where

$$
\bar{\beta}=\frac{-\frac{u_{d}\left(y_{d}^{*}\right)}{y_{e}^{*}}+\sqrt{\left(\frac{u_{d}\left(y_{d}^{*}\right)}{y_{e}^{*}}\right)^{2}+4 \frac{1-\pi_{d} p_{d}}{\left(\pi_{d} p_{d}\right)^{2}}}}{2 \frac{1-\pi_{d} p_{d}}{\pi_{d} p_{d}}} .
$$

Then if $\sigma$ is sufficiently small, the cyclical allocation $\left(x^{+}, y^{*}\right)$, for $x^{+}$in a neighborhood of $x$, is implementable.

Proof. The cutoff value $\bar{\beta}$ was constructed so that $\left(x, y^{*}\right)$ is implementable for $\beta=\bar{\beta}$. Since the participation-constraint sets vary continuously with $(\sigma, \tau)$, the result follows.

\section{Optimal policies}

On one hand, our results regarding extensive-margin effects show that there exists a cutoff value for $\pi_{d}$, called $\bar{\pi}$, such that the maximizer of the average extensive margin is cyclical if and only if $\pi_{d}<\bar{\pi}$. On the other hand, our results on intensive margins show that there exists a cutoff value of $\beta$, called $\bar{\beta}$, such that for $\beta>\bar{\beta}$, small interventions around the allocation $\left(x, y^{*}\right)$, where $p_{s}=q_{s}$, are implementable. If follows, therefore, that the optimum is cyclical for a large set of parameters, including those $\pi_{s}$ and $\beta$ such that $\pi_{d}<\bar{\pi}$ and $\beta>\bar{\beta}$.

Proposition 4 If $\pi_{d}<\bar{\pi}$ and $\beta \geq \bar{\beta}$, then the optimum monetary policy is cyclical. Proof. Welfare is proportional to $\sum_{s} E_{s} I_{s}$, where $E_{s}$ is the extensive margin at $s$, $\pi_{s} p_{s} q_{s}$, and $I_{s}$ is the intensive margin at $s, u_{s}\left(y_{s}\right)-y_{s}$. By lemma $1, E_{e}=E_{d}$ for 
all acyclical policies, so that for fixed $\left(I_{e}, I_{d}\right)$, the acyclical $x$ that maximizes welfare features $p_{s}=q_{s}$. Since $\beta \geq \bar{\beta}$, then $y^{*}$ satisfies participation constraints evaluated at this maximizer, so that the allocation that attains the highest welfare among acyclical policies is $\left(x, y^{*}\right)$. Since a small intervention increases $E_{e}$ and $E_{e}+E_{d}$ when $\pi_{d}<\bar{\pi}$, and $I_{e} \geq I_{d}$ for $y=y^{*}$, and such intervention is implementable according to our last proposition, then the optimal cannot be acyclical.

The proof of this proposition holds even when $u_{e}=u_{d}$. When $u_{e}=u_{d}, \pi_{d}=\bar{\pi}$ and $\beta=\bar{\beta}$, proposition 4 implies that

$$
\sum_{s} \pi_{s} p_{s}^{+} q_{s}^{+}\left[u_{s}\left(y_{s}^{+}\right)-y_{s}^{+}\right]=\sum_{s} \pi_{s} p_{s} q_{s}\left[u_{s}\left(y_{s}^{*}\right)-y_{s}^{*}\right]
$$

where $x^{+}$is a cyclical distribution, $x$ is the (optimal) acyclical distribution and, by construction, $y_{e}^{+}=y_{d}^{+}=y_{d}^{*}=y_{e}^{*}$. However, if $u_{e}^{\prime}>u_{d}^{\prime}, \pi_{d}=\bar{\pi}$ and $\beta=\bar{\beta}$, then

$$
\sum_{s} \pi_{s} p_{s}^{+} q_{s}^{+}\left[u_{s}\left(y_{s}^{+}\right)-y_{s}^{+}\right]>\sum_{s} \pi_{s} p_{s} q_{s}\left[u_{s}\left(y_{s}^{*}\right)-y_{s}^{*}\right]
$$

since $u_{e}\left(y_{e}^{+}\right)-y_{e}^{+}=u_{e}\left(y_{e}^{*}\right)-y_{e}^{*}>u_{d}\left(y_{d}^{+}\right)-y_{d}^{+}=u_{d}\left(y_{d}^{*}\right)-y_{d}^{*}$ and $\pi_{e} p_{e}^{+} q_{e}^{+}>\pi_{e} p_{e}^{*} q_{e}^{*}$ and $\sum_{s} \pi_{s} p_{s}^{+} q_{s}^{+}=\sum_{s} \pi_{s} p_{s} q_{s}$. Therefore, when $u_{e}^{\prime}>u_{d}^{\prime}$ there exists (non-unique) numbers $\hat{\beta}<\bar{\beta}$ and $\hat{\pi}<\bar{\pi}$ such that for any $\beta \in(\hat{\beta}, \bar{\beta})$ and $\pi \in(\hat{\pi}, \bar{\pi})$ inequality (15) holds. Therefore, proposition 4 describes the sufficient, but not necessary conditions, for the optimal money policy to be cyclical. (We have documented these properties with numerical simulations, which are available upon request.) As a result, cyclical monetary policy may be optimal for some economies where the conditions of proposition 4 do not hold.

\section{Conclusion}

We have constructed a random matching model of seasons, where different seasons are characterized by both differing desires and intensities of the buyer to consume. Even when buyer's intensity to consume is constant over seasons - and only the desire to consume varies over seasons - we show that a monetary policy that injects money into the economy when the desire to consume is high and withdraws it when the desire is low may be beneficial. A cyclical policy increases the chances of single 
coincidence meetings in the high season and decreases their chances in the low season, compared to a constant monetary policy. A cyclical policy will be beneficial if the proportion of consumers who want to consume is small in the low season relative to the high season. In this situation the average number of successful matches over both seasons will increase - which in turn increases welfare-because the measure of single coincidence matches in the high season is weighted by a larger factor than that in the low season. When the seasons are characterized by both differing desires and intensities to consume by the buyer, then a cycle monetary policy can be optimal even when the difference between the proportions of the buyers that want to consume in the high and low seasons is not very large. Our theory provides some additional support for the founding of the Fed. Previous explanations relied on the reduction in financial panics that came about after the founding of the Fed; ours relies on the improved production and consumption allocations that result when the Fed follows a cyclical policy that alters amount of money in the economy on a seasonal basis. 


\section{References}

Bewley, T. 1980 "The Optimum Quantity of Money," in J. Kareken and N. Wallace, eds., Models of Monetary Economics (Federal Reserve Bank of Minneapolis, Minneapolis, MN).

Cavalcanti, R. de O. 2004 "A Monetary Mechanism for Sharing Capital: Diamond and Dybvig Meet Kiyotaki and Wright," Economic Theory 24 769-88.

Cavalcanti, R. de O. and N. Wallace 1999 "A Model of Private Banknote Issue," Review of Economic Dynamics 2 104-36.

Deviatov, A. and N. Wallace 2001 "Another Example in which Money Creation is Beneficial," Advances in Macroeconomics 1 article 1.

Friedman, M. 1969 "The Optimum Quantity of Money," in The Optimum Quantity of Money and Other Essays (Aldine, Chicago IL).

Kiyotaki, N. and R. Wright 1989 "On Money as a Medium of Exchange," Journal of Political Economy 91 927-54.

Levine, D. K. 1991 “Asset Trading Mechanisms and Expansionary Policy," Journal of Economic Theory 54 148-64.

Li, V. 1995 "Optimal Taxation of Fiat Money in Search Equilibrium," International Economic Review 36 927-42.

Lucas, R. E. 1972 "Expectations and the Neutrality of Money," Journal of Economic Theory 4 103-24.

Meltzer, A. 2003 A History of the Federal Reserve, Volume 1: 1913-1951 (University of Chicago Press, Chicago IL).

Miron J. 1986 "Financial Panics, the Seasonality of the Nominal Interest Rate and the Founding of the Fed," American Economic Review 76 125-40.

Molico, M. 1999 "Distribution of Money and Prices in Search Equilibrium," unpublished mimeo.

Sheinkman, J. A. and L. Weiss 1986 "Borrowing Constraints and Aggregate Economic Activity," Econometrica 54 23-45.

Shi, S. 1999 "Money, Capital and Redistributive Effects of Monetary Policies," Journal of Economic Dynamics and Control 23 565-90. 


\section{Appendix 1}

Lemma 4 There exists, for each $q_{s}$, an unique acyclical $x$. Moreover, $x$ can be solved for analytically. The statement holds for any $s$ in $\{e, d\}$.

Proof. We shall make repeated used of the system (1-4) with $\sigma=\tau=0$. According to lemma $2, p_{s}=q_{s}+a$ for some $a$ that does not depend on $s$. We shall first solve for $a$ analytically. For this purpose, let $A \equiv 1+\pi_{s} p_{s} q_{s}$, which, by force of lemma 1 , does not depend on $s$ as well. Equation (1) now reads (i) $p_{e}=A-q_{d}$. Using (ii) $p_{d}=q_{d}+a$, we can write $(2)$ as (iii) $q_{e}=A-\left(a+q_{d}\right)$. The equality $p_{e} q_{e}=\theta p_{d} q_{d}$ for $\theta=\pi_{d} / \pi_{e}$, can be written, using (i), (ii) and (iii), as $\left(A-q_{d}\right)^{2}-a\left(A-q_{d}\right)-$ $\theta q_{d}\left(a+q_{d}\right)=0$. The only relevant solution of this quadratic equation is given by (iv) $2\left(A-q_{d}\right)=a+\sqrt{a^{2}+4 \theta b}$, where $b=q_{d}\left(a+q_{d}\right)$. Since $A=1+\pi_{d} q_{d} p_{d}=1+\pi_{d} b$, we can rewrite (iv) as (v) $a^{2}+4 \theta b=\left[2 \pi_{d} b+2\left(1-q_{d}\right)-a\right]^{2}$. Expanding now (v) as a quadratic equation in $b$, we find that the only relevant solution is given by (vi) $2 \pi_{d}^{2} b=\theta+a \pi_{d}-2 \pi_{d}\left(1-q_{d}\right)+\sqrt{\theta^{2}-4 \pi_{d}\left(1-q_{d}\right) \theta+\pi_{d}^{2} a^{2}+2 \theta \pi_{d} a}$. Substituting in (vi) the expression $b=q_{d}\left(a+q_{d}\right)$, produces a quadratic equation in $a$ as a function of $q_{d}$. The only relevant solution of the latter is (vii) $a=\left[-k_{2}-\sqrt{k_{2}^{2}-4 k_{1} k_{3}}\right] /\left(2 k_{1}\right)$, where $k_{1}=\pi_{d}^{2}\left[\left(2 \pi_{d} q_{d}-1\right)^{2}-1\right], k_{2}=2 \pi_{d}\left\{\left(2 \pi_{d} q_{d}-1\right)\left[2\left(\pi_{d} q_{d}\right)^{2}+2 \pi_{d}\left(1-q_{d}\right)-\theta\right]-\theta\right\}$ and $k_{3}=\left[2\left(\pi_{d} q_{d}\right)^{2}+2 \pi_{d}\left(1-q_{d}\right)-\theta\right]^{2}-\theta^{2}+4 \pi_{d}\left(1-q_{d}\right) \theta$. If $q_{d}$ is fixed, then $p_{d}=q_{d}+a$ determines $p_{d}$. Using now (1) and $p_{e}=q_{e}+a$, the values of $p_{e}$ and $q_{e}$ are also determined. Since the system (1-4) is symmetric in $e$ and $d$, when $\sigma=\tau=0$, similar conclusions follows when $q_{e}$ is given, instead of $q_{d}$.

\section{Appendix 2}

Proposition 1 The maximizer of $\pi_{s} p_{s} q_{s}$, among the set of acyclical distributions, is the unique $x$ such that $p_{s^{\prime}}=q_{s^{\prime}}$. The statement holds for any $s$ and $s^{\prime}$ in $\{e, d\}$.

Proof. The set of acyclical distributions is closed, and $\pi_{s} p_{s} q_{s}$ is continuous in $x$ for each $s$, so that a maximizer exists. Let us fix $x=x^{1}$, with $p_{s}^{1} \neq q_{s}^{1}$ for some $s$, and show that $x^{1}$ cannot be the maximizer. Note that, by lemma $2, p_{s}^{1} \neq q_{s}^{1}$ if and only if $p_{s^{\prime}}^{1} \neq q_{s^{\prime}}^{1}$. We start by constructing $x^{2}$, the "mirror image" of $x^{1}$, with the equalities $p_{s}^{2}=q_{s}^{1}$ and $q_{s}^{2}=p_{s}^{1}$ for $s \in\{e, d\}$. Also, for $\alpha \in(0,1)$, let $x^{\alpha} \equiv \alpha x^{1}+(1-\alpha) x^{2}$. 
It is clear that, for all $s, \alpha p_{s}^{1} q_{s}^{1}+(1-\alpha) p_{s}^{2} q_{s}^{2}<p_{s}^{\alpha} q_{s}^{a}$. Thus the distribution $x^{\alpha}$ attains a higher extensive margin than that of $x^{1}$, although $x^{\alpha}$ is not invariant if it does not satisfies (1-4) with equality. However, using now lemma 1, one can rewrite each equation in the system (1-4), when $\sigma=\tau=0$, as $p_{s}+q_{s^{\prime}}=1+\pi_{s} p_{s} q_{s}$ or $p_{s^{\prime}}+q_{s}=1+\pi_{s} p_{s} q_{s}$ where $s^{\prime} \neq s$, so that each right-hand side is increasing in the extensive margin. Since $p_{s}^{\alpha}+q_{s^{\prime}}^{\alpha}<1+\pi_{s} p_{s}^{\alpha} q_{s}^{\alpha}$ and $p_{s^{\prime}}^{\alpha}+q_{s}^{\alpha}<1+\pi_{s} p_{s}^{\alpha} q_{s}^{\alpha}$, then there exists an acyclical $\bar{x}$, with $\bar{x} \geq x^{\alpha}$, that attains a higher extensive margin than that of $x$. The proof is now complete.

\section{Appendix 3}

Lemma 7 If $x$ is invariant and $\tau=\phi \sigma$, then $p_{s}-q_{s}=f_{s}(\sigma)$, where $f_{e}(\alpha)=\frac{\phi-\phi \alpha-1}{1+\phi-\phi \alpha}$ and $f_{d}(\alpha)=\frac{\phi+\phi \alpha-1}{1+\phi-\phi \alpha}$.

Proof. The system (1-4) can be rewritten as

$$
\begin{gathered}
\hat{p}_{e}=1-(1-\tau) \hat{q}_{d}+\pi_{d} p_{d} q_{d}, \\
\hat{p}_{d}=1-(1-\sigma) \hat{q}_{e}+\pi_{d} p_{e} q_{e}+\frac{\tau}{1-\tau}, \\
\hat{q}_{e}=1-(1-\tau) \hat{p}_{d}+\pi_{d} p_{d} q_{d}+\frac{\sigma}{1-\sigma}
\end{gathered}
$$

and

$$
\hat{q}_{d}=1-(1-\sigma) \hat{p}_{e}+\pi_{e} p_{e} q_{e}
$$

where $\hat{p}_{e}=p_{e} /(1-\sigma), \hat{p}_{d}=p_{d} /(1-\tau), \hat{q}_{e}=q_{e} /(1-\sigma)$ and $\hat{q}_{d}=q_{d} /(1-\tau)$. Eliminating $\pi_{d} p_{d} q_{d}$ between equations (16) and (18), and $\pi_{e} p_{e} q_{e}$ between (17) and (19), yields

$$
\hat{p}_{e}-\hat{q}_{e}=(1-\tau)\left(\hat{p}_{d}-\hat{q}_{d}\right)-\frac{\sigma}{1-\sigma}
$$

and

$$
\hat{p}_{d}-\hat{q}_{d}=(1-\sigma)\left(\hat{p}_{e}-\hat{q}_{e}\right)+\frac{\tau}{1-\tau},
$$

which can now be solved as

$$
\hat{p}_{e}-\hat{q}_{e}=\frac{(1-\sigma) \tau-\sigma}{(1-\sigma)[1-(1-\tau)(1-\sigma)]}
$$

and

$$
\hat{p}_{d}-\hat{q}_{d}=\frac{\tau-(1-\tau) \sigma}{(1-\tau)[1-(1-\tau)(1-\sigma)]} .
$$


One can now multiply both sides of $(20)$ by $1-\sigma$ to obtain the expression $p_{e}-q_{e}=$ $f_{e}(\sigma)$, and multiply both sides of $(21)$ by $1-\tau$ to obtain the expression $p_{d}-q_{d}=f_{d}(\sigma)$.

\section{Appendix 4}

Before we can provide a proof for proposition 2, the following two lemmas are needed. From lemma 7 we can use the expression $q_{s}=p_{s}-f_{s}$ to reduce (1)-(4) to a system in $\left(p_{e}, p_{d}\right)$, which allows us to write the derivatives of $p_{s}$ with respect to $\sigma$ as follows.

Lemma 8 If $x$ is invariant and $\tau=\phi \sigma$, then the derivatives of $p_{s}$ with respect to $\sigma$, evaluated at $\sigma=0$, satisfy

$$
\left[\begin{array}{cc}
1 & 1-\pi_{d}\left(2 p_{d}-f_{d}\right) \\
1-\pi_{e}\left(2 p_{e}-f_{e}\right) & 1
\end{array}\right]\left[\begin{array}{c}
p_{e}^{\prime} \\
p_{d}^{\prime}
\end{array}\right]=\left[\begin{array}{c}
\left(1-\pi_{d} p_{d}\right) f_{d}^{\prime}-p_{e} \\
\left(1-\pi_{e} p_{e}\right) f_{e}^{\prime}-\phi p_{d}+\phi
\end{array}\right]
$$

Proof. Equations (1) and (2) can be written as

$$
\frac{p_{e}^{+}}{1-\sigma}=1-p_{d}^{+}+f_{d}+E_{d}
$$

and

$$
\frac{p_{d}^{+}}{1-\phi \sigma}-\frac{\phi \alpha}{1-\phi \sigma}=1-p_{e}^{+}+f_{e}+E_{e}
$$

where $E_{d}=\pi_{d} p_{d}^{+}\left(p_{d}^{+}-f_{d}\right)$ and $E_{e}=\pi_{e} p_{e}^{+}\left(p_{e}^{+}-f_{e}\right)$. Taking derivatives on both sides of (22) and (23), with respect to $\sigma$, yields, for $\sigma=0$,

$$
p_{e}+p_{e}^{\prime}=-p_{d}^{\prime}+f_{d}^{\prime}+E_{d}^{\prime}
$$

and

$$
\phi p_{d}+p_{d}^{\prime}-\phi=-p_{e}^{\prime}+f_{e}^{\prime}+E_{e}^{\prime}
$$

where $E_{d}^{\prime}=\pi_{d} p_{d}^{\prime}\left(2 p_{d}-f_{d}\right)-\pi_{d} p_{d} f_{d}^{\prime}$ and $E_{e}^{\prime}=\pi_{e} p_{e}^{\prime}\left(2 p_{e}-f_{e}\right)-\pi_{e} p_{e} f_{e}^{\prime}$. Substituting the expressions for $E_{d}^{\prime}$ and $E_{e}^{\prime}$ into equations (24) and (25), yields the result.

The total effect of changes in $\sigma$ on extensive margins can also be expressed in a compact form.

Lemma 9 If $x$ is invariant and $\tau=\phi \sigma$, then the derivative of the sum $\sum_{s} \pi_{s} p_{s} q_{s}$, with respect to $\sigma$, evaluated at $\sigma=0$, is equal to $p_{e}+\phi p_{d}-\phi-f_{e}^{\prime}-f_{d}^{\prime}+2\left(p_{e}^{\prime}+p_{d}^{\prime}\right)$. 
Proof. Using equations (24) and (25), derived in the proof of the previous lemma, yields the results, since the derivative of the sum $\sum_{s} \pi_{s} p_{s} q_{s}$ is precisely $E_{d}^{\prime}+E_{e}^{\prime}$.

Using now the last lemmas 7, 8 and 9, we can characterize the sign of the derivative of the sum $\sum_{s} \pi_{s} p_{s} q_{s}$, for $p_{s}=q_{s}$, as follows.

Proposition 2 The maximizer of sum $\sum_{s} \pi_{s} p_{s} q_{s}$ is cyclical if and only if $\pi_{d} \in[0, \bar{\pi}]$, where $\bar{\pi} \in\left(0, \pi_{e}\right)$ can be solved for analytically as a function of $\pi_{e}$.

Proof. The last three lemmas allow the substitution of expressions for $p_{e}^{\prime}+p_{d}^{\prime}$ and $f_{e}^{\prime}+f_{d}^{\prime}$ into the expression of the derivative of $\sum_{s} \pi_{s} p_{s} q_{s}$, evaluated at $\sigma=0, p_{s}=q_{s}$ and $\phi=1$. Substituting also the analytical solution for $p_{e}$ and $p_{d}$, when $p_{s}=q_{s}$ and $\sigma=0$ from lemma 5 , yields an expression for the derivative involving only parameters. After some tedious but straightforward algebra, the condition according to which this derivative is positive can be written as

$$
2 \pi_{d} \leq(1-\theta) \sqrt{2}-(1-\sqrt{\theta})^{2}
$$

where $\theta=\pi_{d} / \pi_{e}$. The inequality is not satisfied for $\theta=1$ and $\pi_{d}>0$. Hence the cutoff value of $\pi_{d}$ for which the derivative is positive must be below $\pi_{e}$. Imposing now equality in this expression and substituting for the value of $\theta$ yields, after solving for the unique relevant solution of the implied quadratic equation in $\pi_{d}^{2}$,

$$
\bar{\pi}=\frac{1}{4}\left[\frac{2 / \sqrt{\pi_{e}}+\sqrt{4 / \pi_{e}-4\left(2+(1+\sqrt{2}) / \pi_{e}\right)(1-\sqrt{2})}}{2+(1+\sqrt{2}) / \pi_{e}}\right]^{2},
$$

which has the properties stated in the proposition.

\section{Appendix 5}

Before providing a proof to proposition 3 , we will first rewrite $(v, w)$ in a convenient form and then will introduce two lemmas that will be needed in proof. Substituting the values of $(\bar{v}, \bar{w})$ from equation (6) into equation (5), allows us to work with two independent systems of Bellman equations in $(v, w)$, represented in matrix format as

$$
\left[\begin{array}{c}
v_{s} \\
w_{s^{\prime}}
\end{array}\right]=\frac{1}{\operatorname{det}\left(M_{s s^{\prime}}\right)} M_{s s^{\prime}}\left[\begin{array}{c}
\mu_{u s} \pi_{s} p_{s} u_{s} \\
-\mu_{y s^{\prime}} \pi_{s^{\prime}} q_{s^{\prime}} y_{s^{\prime}}
\end{array}\right]
$$

where $s, s^{\prime} \in\{e, d\}, s^{\prime} \neq s, \mu_{u e}=\mu_{y d}=1, \mu_{u d}=1-\tau, \mu_{y e}=1-\sigma$, and

$$
M_{s s^{\prime}}=\left[\begin{array}{cc}
1-\left(1-\pi_{s^{\prime}} q_{s^{\prime}}\right) \delta(1-\sigma) & \tau \beta+(1-\tau) \pi_{s} p_{s} \beta \\
\sigma \beta+(1-\sigma) \pi_{s^{\prime}} q_{s^{\prime}} \beta & 1-\left(1-\pi_{s} p_{s}\right) \delta(1-\tau)
\end{array}\right] .
$$


We start with the following lemma that allow us to ignore $\operatorname{det}\left(M_{s s^{\prime}}\right)$ in the algebra that follows.

Lemma 8 The determinant of $M_{s s^{\prime}}$ is positive.

Proof. For $a_{d} \equiv 1-\pi_{d} q_{d}$ and $a_{e} \equiv 1-\pi_{e} p_{e}$, the determinant of $M_{e d}$ equals

$$
\left(1-\delta a_{d}+\sigma \delta a_{d}\right)\left(1-\delta a_{e}+\tau \delta a_{e}\right)-\delta\left(\pi_{d} q_{d}+\sigma a_{d}\right)\left(\pi_{e} p_{e}+\tau a_{e}\right)
$$

which can be written as the sum of two terms, $k_{0}$ and $k_{1}$, where $k_{0}$ contains all the terms without $\sigma$ or $\tau$, and $k_{1}$ contains the other terms. The expression for $k_{0}$ is

$$
k_{0}=\left[1-\delta\left(1-\pi_{d} q_{d}\right)\right]\left[1-\delta\left(1-\pi_{e} q_{e}\right)\right]-\delta \pi_{d} q_{d} \pi_{e} q_{e} .
$$

After some simple algebra, that expression becomes

$$
k_{0}=(1-\delta)\left(1-\delta+\delta \pi_{d} q_{d}+\delta \pi_{e} q_{e}-\delta \pi_{d} q_{d} \pi_{e} q_{e}\right)
$$

which is positive if $x$ is invariant. Likewise, since for $a_{d} \equiv 1-\pi_{d} q_{d}$ and $a_{e} \equiv 1-\pi_{e} p_{e}$ one can write $k_{1}$ as

$$
\begin{aligned}
& \tau \delta a_{e}\left(1-\delta a_{d}-\pi_{d} q_{d}\right)+\sigma \delta a_{d}\left(1-\delta a_{e}-\pi_{e} p_{e}\right)+\delta \sigma a_{d} \tau a_{e}(\delta-1), \text { or } \\
& \tau \delta a_{e}(1-\delta)\left(1-\pi_{d} q_{d}\right)+\sigma \delta a_{d}(1-\delta)\left(1-\pi_{e} p_{e}\right)-\sigma \delta a_{d}(1-\delta) \tau a_{e}, \text { or } \\
& \tau \delta a_{e}(1-\delta)\left(1-\pi_{d} q_{d}\right)+\sigma \delta a_{d}(1-\delta)\left(1-\pi_{e} p_{e}\right)(1-\tau),
\end{aligned}
$$

which is nonnegative. A similar argument shows that $\operatorname{det}\left(M_{d e}\right)$ is also positive.

Next, we use the Bellman equation for $w_{e}$ to write $(7)$ in an equivalent format that does not depend on $y_{e}$ explicitly.

Lemma 9 The participation constraint for date-s producers is equivalent to [1-(1$\sigma) \delta] w_{s} \geq \sigma \beta v_{s^{\prime}}$.

Proof. Let $s=e$. The Bellman equation for $w_{e}$ can be written as

$$
[1-(1-\sigma) \delta] w_{e}-\sigma \beta v_{d}=(1-\sigma) \pi_{e} q_{e}\left(-y_{e}+\beta v_{d}-\beta \bar{v}_{d}\right)
$$

then the result follows directly from (7). The argument for $s=d$ also follows from the same steps. 
We now use the previous two lemmas to write the slack in the producer constraint in matrix algebra as

$$
\left[\begin{array}{ll}
-\sigma \beta & 1-(1-\sigma) \delta
\end{array}\right]\left[\begin{array}{c}
v_{s} \\
w_{s^{\prime}}
\end{array}\right]=\frac{1}{\operatorname{det}\left(M_{s s^{\prime}}\right)}\left[\begin{array}{ll}
m_{u s} & m_{y s^{\prime}}
\end{array}\right]\left[\begin{array}{c}
\mu_{u s} \pi_{s} p_{s} u_{s} \\
-\mu_{y s^{\prime}} \pi_{s^{\prime}} q_{s^{\prime}} y_{s^{\prime}}
\end{array}\right]
$$

where the scalars $m_{u s}$ and $m_{y s^{\prime}}$ are to be computed, so that the sign of the participation constraint does not depend on the magnitude of $\operatorname{det}\left(M_{s s^{\prime}}\right)$. After some straightforward algebra is used to produce a simple expression for $m_{u s}$ and $m_{y s^{\prime}}$, the desired inequalities are derived as follows.

Proposition 3 The participation constraints are satisfied if and only if

$$
u_{d}\left(y_{d}\right) \geq \frac{y_{e}}{\beta}\left[\frac{1}{(1-\tau) \pi_{d} p_{d}}-(1-\sigma) \delta \frac{1-\pi_{d} p_{d}}{\pi_{d} p_{d}}\right]
$$

and

$$
u_{e}\left(y_{e}\right) \geq \frac{y_{d}}{\beta}\left[\frac{1}{(1-\sigma) \pi_{e} p_{e}}-(1-\tau) \delta \frac{1-\pi_{e} p_{e}}{\pi_{e} p_{e}}\right]
$$

Proof. The steps for deriving the inequality (28) are simple; we omit the proof for inequality (29) it is identical to the proof of inequality (28). Regarding participation constraint for date- $e$ producers, we find it useful to set $\rho=\pi_{d} p_{d}$ and $\xi=\pi_{e} q_{e}$ so that the expression for $m_{u d}$ can be written as

$$
\begin{aligned}
-m_{u d}= & \sigma \beta-\sigma \beta(1-\sigma)(1-\xi) \delta-\sigma \beta-(1-\sigma) \xi \beta+\sigma \beta(1-\sigma) \delta+ \\
& (1-\sigma) \xi \beta(1-\sigma) \delta \\
= & -(1-\sigma) \xi \beta+(1-\sigma) \xi \beta \delta \\
= & -(1-\delta)(1-\sigma) \xi \beta .
\end{aligned}
$$


That for $m_{y e}$ is

$$
\begin{aligned}
-m_{y e}= & \sigma \beta \tau \beta+\sigma \beta(1-\tau) \rho \beta-1+(1-\tau)(1-\rho) \delta+(1-\sigma) \delta+ \\
& -(1-\sigma) \delta(1-\tau)(1-\rho) \delta \\
= & \sigma \beta \tau \beta+\sigma(1-\tau) \rho \delta-1+(1-\sigma) \delta+ \\
& -(1-\tau)(1-\rho) \delta[(1-\sigma) \delta-1] \\
= & -1+\delta-\sigma \delta[1-\tau-(1-\tau) \rho]+\sigma \delta(1-\tau)(1-\rho) \delta+ \\
& (1-\delta)(1-\tau)(1-\rho) \delta \\
= & -1+\delta-\sigma \delta(1-\tau)(1-\rho)+\sigma \delta(1-\tau)(1-\rho) \delta+ \\
& (1-\delta)(1-\tau)(1-\rho) \delta \\
= & -1+\delta-(1-\delta) \sigma \delta(1-\tau)(1-\rho)+(1-\delta)(1-\tau)(1-\rho) \delta \\
= & -(1-\delta)[1-(1-\sigma) \delta(1-\tau)(1-\rho)]
\end{aligned}
$$

Thus, the right-hand side of (27) equals

$$
\frac{(1-\delta)(1-\sigma) \pi_{e} q_{e}}{\operatorname{det}\left(M_{d e}\right)}\left[\begin{array}{ll}
\beta & 1-\delta(1-\sigma)(1-\tau)\left(1-\pi_{d} p_{d}\right)
\end{array}\right]\left[\begin{array}{c}
(1-\tau) \pi_{d} p_{d} u_{d} \\
-y_{e}
\end{array}\right],
$$

so that (28) follows. 


\section{Últimos Ensaios Econômicos da EPGE}

[553] Aloisio Pessoa de Araújo, Daniel Gottlieb, e Humberto Luiz Ataide Moreira. A model of mixed signals with applications to countersignaling an the GED. Ensaios Econômicos da EPGE 553, EPGE-FGV, Jul 2004.

[554] Carlos Eugênio Ellery Lustosa da Costa e Lucas Jóver Maestri. The riskproperties of human capital and the design of government policies. Ensaios Econômicos da EPGE 554, EPGE-FGV, Jul 2004.

[555] Daniel Gottlieb e Lucas Jóver Maestri. Banning information as a redistributive device. Ensaios Econômicos da EPGE 555, EPGE-FGV, Jul 2004.

[556] Leonardo Pio Perez e Pedro Cavalcanti Gomes Ferreira. Efeitos macroeconômicos e custos sociais de uma transição entre regimes de previdência no Brasil. Ensaios Econômicos da EPGE 556, EPGE-FGV, Jul 2004.

[557] Rubens Penha Cysne. Inflation and income inequality: A link through the jobsearch process. Ensaios Econômicos da EPGE 557, EPGE-FGV, Ago 2004.

[558] Rubens Penha Cysne. A search-theoretic explanation for the negative correlation between labor income and impatience. Ensaios Econômicos da EPGE 558, EPGE-FGV, Ago 2004.

[559] Rubens Penha Cysne. Income inequality:The role of impatience in a job-search process. Ensaios Econômicos da EPGE 559, EPGE-FGV, Ago 2004.

[560] Rubens Penha Cysne. Towards a measure of income inequality freed from the volatility caused by variations in the rate of unemployment. Ensaios Econômicos da EPGE 560, EPGE-FGV, Ago 2004.

[561] Rubens Penha Cysne. On the positive correlation between income inequality and unemployment. Ensaios Econômicos da EPGE 561, EPGE-FGV, Ago 2004.

[562] Rubens Penha Cysne. A general-equilibrium closed-form solution to the welfare costs of inflation (Forthcoming, Revista Brasileira de Economia). Ensaios Econômicos da EPGE 562, EPGE-FGV, Ago 2004.

[563] Marcelo Côrtes Neri e Marcelo Casal Xerez. Aspectos dinâmicos de um sistema de metas sociais. Ensaios Econômicos da EPGE 563, EPGE-FGV, Ago 2004.

[565] Marcelo Côrtes Neri e Marcelo Casal Xerez. Desenho de um sistema de metas sociais. Ensaios Econômicos da EPGE 565, EPGE-FGV, Set 2004.

[566] Rubens Penha Cysne, Wilfredo Maldonado, e Paulo Klinger Monteiro. Inflation and Income Inequality: A Shopping-Time Aproach (Forthcoming, Journal of Development Economics). Ensaios Econômicos da EPGE 566, EPGE-FGV, Set 2004. 
[567] Rubens Penha Cysne. Solving the Non-Convexity Problem in Some ShoppingTime and Human-Capital Models. Ensaios Econômicos da EPGE 567, EPGEFGV, Set 2004.

[568] Paulo Klinger Monteiro. First-Price auction symmetric equlibria with a general distribution. Ensaios Econômicos da EPGE 568, EPGE-FGV, Set 2004.

[569] Pedro Cavalcanti Ferreira, Samuel de Abreu Pessôa, e Fernando A. Veloso. On The Tyranny of Numbers: East Asian Miracles in World Perspective. Ensaios Econômicos da EPGE 569, EPGE-FGV, Out 2004.

[570] Rubens Penha Cysne. On the Statistical Estimation of Diffusion Processes A Partial Survey (Revised Version, Forthcoming Brazilian Review of Econometrics). Ensaios Econômicos da EPGE 570, EPGE-FGV, Out 2004.

[571] Aloisio Pessoa de Araújo, Luciano I. de Castro Filho, e Humberto Luiz Ataide Moreira. Pure strategy equilibria of multidimensional and Non-monotonic auctions. Ensaios Econômicos da EPGE 571, EPGE-FGV, Nov 2004.

[572] Rubens Penha Cysne e Paulo César Coimbra Lisbôa. Imposto Inflacionário e Transferências Inflacionárias no Mercosul e nos Estados Unidos. Ensaios Econômicos da EPGE 572, EPGE-FGV, Nov 2004.

[573] Renato Galvão Flôres Junior. Os desafios da integração legal. Ensaios Econômicos da EPGE 573, EPGE-FGV, Dez 2004.

[574] Gustavo M. de Athayde e Renato Galvão Flôres Junior. Do Higher Moments Really Matter in Portfolio Choice?. Ensaios Econômicos da EPGE 574, EPGEFGV, Dez 2004.

[575] Germán Calfat e Renato Galvão Flôres Junior. The EU-Mercosul free trade agreement: Quantifying mutual gains. Ensaios Econômicos da EPGE 575, EPGE-FGV, Dez 2004.

[576] Andrew W. Horowitz e Renato Galvão Flôres Junior. Beyond indifferent players: On the existence of Prisoners Dilemmas in games with amicable and adversarial preferences. Ensaios Econômicos da EPGE 576, EPGE-FGV, Dez 2004.

[577] Rubens Penha Cysne. Is There a Price Puzzle in Brazil? An Application of Bias-Corrected Bootstrap. Ensaios Econômicos da EPGE 577, EPGE-FGV, Dez 2004.

[603] Ricardo de O. Cavalcanti e Ed Nosal. Some Benets of Cyclical Monetary Policy. Ensaios Econômicos da EPGE 603, EPGE-FGV, Out 2005. 